January 11, 2021 18:56 WSPC/INSTRUCTION FILE Duplij-partHam-ijggmparxiv

International Journal of Geometric Methods in Modern Physics (c) World Scientific Publishing Company

\title{
Formulation of singular theories in a partial Hamiltonian formalism using a new bracket and multi-time dynamics
}

\author{
Steven Duplij \\ Mathematisches Institut, Universität Münster \\ Einsteinstr. 62, D-48149 Münster, Germany \\ duplijs@math.uni-muenster.de
}

Received (6 August, 2013)

Accepted (20 August 2014)

Published

\begin{abstract}
A formulation of singular classical theories (determined by degenerate Lagrangians) without constraints is presented. A partial Hamiltonian formalism in the phase space having an initially arbitrary number of momenta (which can be smaller than the number of velocities) is proposed. The equations of motion become first-order differential equations, and they coincide with those of multi-time dynamics, if a certain condition is imposed. In a singular theory, this condition is fulfilled in the case of the coincidence of the number of generalized momenta with the rank of the Hessian matrix. The noncanonical generalized velocities satisfy a system of linear algebraic equations, which allows an appropriate classification of singular theories (gauge and nongauge). A new antisymmetric bracket (similar to the Poisson bracket) is introduced, which describes the time evolution of physical quantities in a singular theory. The origin of constraints is shown to be a consequence of the (unneeded in our formulation) extension of the phase space, when the new bracket transforms into the Dirac bracket. Quantization is briefly discussed.
\end{abstract}

Keywords: Dirac constraints; degenerate Lagrangian; Hessian; Legendre transform; Poisson bracket; multi-time dynamics.

Mathematics Subject Classification 2010: 37J05, 44A15, 70H45

\section{Contents}

1 Introduction

2 Preliminaries $\quad 2$

3 Partial Hamiltonian formalism

4 Singular theories

5 Origin of constraints in singular theories

6 Conclusion 14

A Appendix: Singular theory as multi-time dynamics 14 


\section{Introduction}

Many modern physical models are gauge theories (see, for example, [1]), which are described at the classical level by singular (degenerate) Lagrangians [2]. The transition to the normally used sequential quantum Hamiltonian formalism for such singular theories is non-trivial (because it is not possible to directly apply the Legendre transformation [3, 4]), and requires additional constructions [56]. The main difficulty lies in the appearance of additional relations between the dynamical variables, which are called constraints [7]. These are used to construct a reduced phase space with fewer positions and corresponding momenta. Further, the selection of the physical subspace of the reduced phase space), where one can consistently carry out the procedure of quantization is needed. [8, 9]. Despite the widespread use of constraint theory [10,11], it is not free from internal contradictions and problems [12,13]. So it makes sense to revise the Hamiltonian formalism itself for singular theories with degenerate Lagrangians [14, 15].

The purpose of this paper is to describe singular theories without the help of constraints. Firstly, a partial Hamiltonian formalism for any Lagrangian system is constructed with an arbitrary number of momenta which can be less than the number of velocities. The corresponding system of equations of motion derived from the principle of least action contains the first derivatives of the canonical variables and second-order derivatives of the generalized noncanonical coordinates (for their velocities we do not define corresponding momenta). Under certain conditions, the equations for the latter are differential-algebraic equations of first order; such a physical system is equivalent to a multi-time dynamics (see Appendix). These conditions exist in theories with degenerate Lagrangians, if the number of momenta is equal to the rank of the Hessian. Then the equations for the noncanonical generalized velocities are algebraic, and the dynamics is defined in terms of new bracket, which, like the Poisson bracket, is antisymmetric and satisfies the Jacobi identity. In this formalism, there are no additional relations between the dynamical variables (constraints). It is shown that, if we extend the phase space so that additional ("extra") momenta corresponding to the noncanonical generalized velocities are defined, the constraints would appear, a new bracket turns into the Dirac bracket, and suitable formulas reproduce the Dirac theory [7]. For clarity, we use local coordinates and consider a system with a finite number of degrees of freedom.

\section{Preliminaries}

A dynamical system (with a finite number of degrees of freedom) can be defined in terms of generalized (Lagrangian) coordinates $q^{A}(t), A=1, \ldots, n$ (as a function of time $t$ ) in the configuration space $Q_{n}$ (we write its dimension $n$ as a lower index). The trajectory in the configuration space $Q_{n}$, is determined by the equations of motion, which is a set of differential equations for the generalized coordinates $q^{A}(t)$, and their time derivatives $\dot{q}^{A}(t)$, where $\dot{q}^{A}(t) \equiv d q^{A}(t) / d t$, which define the tangent bundle $T Q_{n}$ of rank $n$ (so that the dimension of the total space is $2 n$ ) [16]. Here we do not consider systems with higher derivatives (see, e.g., [17, 18]). The equations of motion can be obtained using different principles of action, which identify the actual trajectory with the requirement 
of a functional extremity [19]. The standard principle of least action [19] considers the functional

$$
S=\int_{t_{0}}^{t} L d t^{\prime}
$$

where a differentiable function $L=L\left(t, q^{A}, \dot{q}^{A}\right)$ is a Lagrangian, and the functional (on extremals) $S=S\left(t, q^{A}\right)$ is the action of a dynamical system as a function of the upper limit of $t$ (a fixed lower limit $t_{0}$ ). Let us consider an infinitesimal variation of the functional (2.1) $\delta S=S\left(t+\delta t, q^{A}+\delta q^{A}\right)-S\left(t, q^{A}\right)$. Without loss of generality, we can assume that the lower limit of the variation vanishes $\delta q^{A}\left(t_{0}\right)=0$, while the upper denotes the trajectory $\delta q^{A}$. For the variation of $\delta S$ after integration by parts

$$
\delta S=\int_{t_{0}}^{t}\left(\frac{\partial L}{\partial q^{A}}-\frac{d}{d t^{\prime}}\left(\frac{\partial L}{\partial \dot{q}^{A}}\right)\right) \delta q^{A}\left(t^{\prime}\right) d t^{\prime}+\frac{\partial L}{\partial \dot{q}^{A}} \delta q^{A}+\left(L-\frac{\partial L}{\partial \dot{q}^{A}} \dot{q}^{A}\right) \delta t
$$

Then, from the principle of least action of $\delta S=0$, we obtain the equations of motion (Euler-Lagrange equations) [20]

$$
\frac{\partial L}{\partial q^{A}}-\frac{d}{d t}\left(\frac{\partial L}{\partial \dot{q}^{A}}\right)=0 \quad A=1, \ldots, n
$$

which determine the extremals under the condition that all $\delta q^{A}=0$ and $\delta t=0$. The second and third terms in (2.2) define the total differential of the action (on extremals) as a function of $(n+1)$ variables: the coordinates and the upper limit of integration in (2.1)

$$
d S=\frac{\partial L}{\partial \dot{q}^{A}} d q^{A}+\left(L-\frac{\partial L}{\partial \dot{q}^{A}} \dot{q}^{A}\right) d t .
$$

Thus, from the definition of the action (2.1) and (2.4), it follows that $\frac{d S}{d t}=L, \frac{\partial S}{\partial q^{A}}=$ $\frac{\partial L}{\partial \dot{q}^{A}}, \frac{\partial S}{\partial t}=L-\frac{\partial L}{\partial \dot{q}^{A}} \dot{q}^{A}$. In the standard Hamiltonian formalism [20], to each coordinate $q^{A}$ one can assign the canonically conjugate momentum $p_{A}$ by the formula

$$
p_{A}=\frac{\partial L}{\partial \dot{q}^{A}}, \quad A=1, \ldots, n .
$$

If the system of equations (2.5) is solvable for all velocities $\dot{q}^{A}$, we can define the Hamiltonian by the Legendre transform [20]

$$
H=p_{A} \dot{q}^{A}-L,
$$

which defines the mapping between the cotangent and tangent bundles $T Q_{n}^{*} \rightarrow T Q_{n}[16]$. The r.h.s. of (2.6) is expressed in terms of the momenta, so that $H=H\left(t, q^{A}, p_{A}\right)$ is a function on the phase space (or the cotangent bundle $T Q_{n}^{*}$ of rank $n$ and the dimension of the total space $2 n$ ), that is independent of $2 n$ canonical coordinates $\left(q^{A}, p_{A}\right)$. In the standard canonical formalism, each coordinate $q^{A}$ has its conjugate momentum $p_{A}$ by the

${ }^{a}$ Repeated upper and lower indices imply summation. Indices in the function arguments are not to be summed, rather they are written explicitly to distinguish between different types of variables. 
formula (2.5), we call it a full Hamiltonian formalism (in the full phase space $T Q_{n}^{*}$ ). Then the differential of the action (2.4) can also be written as

$$
d S=p_{A} d q^{A}-H d t .
$$

Therefore, for partial derivatives we get

$$
\frac{\partial S}{\partial q^{A}}=p_{A}, \quad \frac{\partial S}{\partial t}=-H\left(t, q^{A}, p_{A}\right)
$$

which implies the Hamilton-Jacobi differential equation

$$
\frac{\partial S}{\partial t}+H\left(t, q^{A}, \frac{\partial S}{\partial q^{A}}\right)=0
$$

which fully determines the dynamics in terms of the given Hamiltonian $H\left(t, q^{A}, p_{A}\right)$. Variation of the action

$$
S=\int\left(p_{A} d q^{A}-H d t\right)
$$

while considering the coordinates and momenta as independent variables, and further integration by parts leads in a standard way [20] to Hamilton's equations in the differential form $b$

$$
d q^{A}=\frac{\partial H}{\partial p_{A}} d t, \quad d p_{A}=-\frac{\partial H}{\partial q^{A}} d t
$$

for the full Hamiltonian formalism (that is, the dynamical system is fully specified by $T Q_{n}^{*}$ ). If we define the (full) Poisson bracket by

$$
\{A, B\}_{\text {full }}=\frac{\partial A}{\partial q^{A}} \frac{\partial B}{\partial p_{A}}-\frac{\partial B}{\partial q^{A}} \frac{\partial A}{\partial p_{A}},
$$

then the equation (2.11) can be written in the standard form [20]

$$
d q^{A}=\left\{q^{A}, H\right\}_{\text {full }} d t, \quad d p_{A}=\left\{p_{A}, H\right\}_{\text {full }} d t .
$$

It is clear that the two formulations of the principle of the least action, that is (2.1) and (2.10), are completely equivalent (they describe the same dynamics) from the definitions of the momenta (2.5) and the Hamiltonian (2.6). Now we will show in this simple language, how to describe the same dynamics using fewer generalized momenta than the number of generalized coordinates.

\section{Partial Hamiltonian formalism}

The transition from a full to a partial Hamiltonian formalism and a multi-time dynamics can be analyzed using the following well-known classical analogy [19], but in its reverse form. In the study of the parametric form of the canonical equations and action $(2.10)$, one

\footnotetext{
${ }^{\mathrm{b}}$ In fact, the equations 2.11 are the conditions for a closed differential 1-form [2.7 (Poincare-Cartan) [16].
} 
formally introduces the extended phase space with the following additional position and momentum

$$
\begin{aligned}
& q^{n+1}=t, \\
& p_{n+1}=-H .
\end{aligned}
$$

Then the action (2.10) takes the symmetrical form and contains only the first term [19] (see, also [21])

$$
S=\int \sum_{A=1}^{n+1} p_{A} d q^{A} .
$$

Here we proceed in the opposite way, and ask: can we, on the contrary, reduce the number of momenta that describe the dynamical system, that is, can we formulate a partial Hamiltonian formalism, which would be equivalent (at the classical level) to the Lagrangian formalism? In other words, is it possible to describe the system with the initial action (2.1) in a smaller phase space initially which is not reduced, because the full phase space is not defined at all. Can we build an analog of the action (2.10) and obtain equations of motion which are equivalent to the Lagrange equations of motion (2.3), and what additional conditions are needed for this? It turns out that the answer to all these questions is positive and leads to a description of singular theories (with degenerate Lagrangians) without introducing constraints [14, 15].

We define a partial Hamiltonian formalism [22], so that the conjugate momentum is associated not to every $q^{A}$ by the formula (2.5), but only for the first $n_{p}<n$ generalized coordinates, which are called canonical and denoted by $q^{i}, i=1, \ldots n_{p}$. The resulting manifold $T Q_{n_{p}}^{*}$ is defined by $2 n_{p}$ (reduced) canonical coordinates $\left(q^{i}, p_{i}\right)$. The rest of the generalized coordinates are called noncanonical $q^{\alpha}, \alpha=n_{p}+1, \ldots n$, and they form a configuration subspace $Q_{n-n_{p}}$, which corresponds to the tangent bundle $T Q_{n-n_{p}}$ (a subscript indicates the corresponding dimension of the total space). Thus, the dynamical system is now given on the direct product (of manifolds) $T Q_{n_{p}}^{*} \times T Q_{n-n_{p}}$. For reduced generalized momenta we have

$$
p_{i}=\frac{\partial L}{\partial \dot{q}^{i}}, \quad i=1, \ldots, n_{p} .
$$

A partial Hamiltonian, similar to (2.6), is defined by a partial Legendre transform

$$
H_{0}=p_{i} \dot{q}^{i}+\frac{\partial L}{\partial \dot{q}^{\alpha}} \dot{q}^{\alpha}-L,
$$

which defines a mapping of $T Q_{n_{p}}^{*} \times T Q_{n-n_{p}} \rightarrow T Q_{n}$ (see (2.6)). In (3.5) the canonical generalized velocities $\dot{q}^{i}$ are expressed in terms of the reduced canonical momenta $p_{i}$ by (3.4). For the action differential (2.7) we can write

$$
d S=p_{i} d q^{i}+\frac{\partial L}{\partial \dot{q}^{\alpha}} d q^{\alpha}-H_{0} d t
$$

We define

$$
H_{\alpha}=-\frac{\partial L}{\partial \dot{q}^{\alpha}}, \quad \alpha=n_{p}+1, \ldots n
$$


and call the functions $H_{\alpha}$ additional Hamiltonians, then

$$
d S=p_{i} d q^{i}-H_{\alpha} d q^{\alpha}-H_{0} d t .
$$

Note that without the second term in (3.8) the partial Hamiltonian (3.5) is the Routh function $R=p_{i} \dot{q}^{i}-L$, in terms of which the Lagrange equations of motion can be reformulated [20]. However, a consistent formulation of the principle of least action for $S$ and a multi-time dynamics of singular systems [14] (see Appendix) is natural in terms of the introduced additional Hamiltonians $H_{\alpha}$ (3.7), while coordinates $q^{\alpha}$ can be treated as additional times (effectively, which can be observed from (3.8)).

Thus, in the partial Hamiltonian formalism the dynamics is completely determined by not one Hamiltonian $H_{0}$ only, but by the set of $\left(n-n_{p}+1\right)$ Hamiltonians $H_{0}, H_{\alpha}$, $\alpha=n_{p}+1, \ldots n$. Indeed, it follows from (3.8) that the partial derivatives of the action $S=S\left(t, q^{i}, q^{\alpha}\right)$ are (see (2.8) for the standard case)

$$
\begin{aligned}
\frac{\partial S}{\partial q^{i}} & =p_{i}, \\
\frac{\partial S}{\partial q^{\alpha}} & =-H_{\alpha}\left(t, q^{i}, p_{i}, q^{\alpha}, \dot{q}^{\alpha}\right), \\
\frac{\partial S}{\partial t} & =-H_{0}\left(t, q^{i}, p_{i}, q^{\alpha}, \dot{q}^{\alpha}\right),
\end{aligned}
$$

which yields the system $\left(n-n_{p}+1\right)$ of Hamilton-Jacobi equations

$$
\begin{aligned}
\frac{\partial S}{\partial t}+H_{0}\left(t, q^{i}, \frac{\partial S}{\partial q^{i}}, q^{\alpha}, \dot{q}^{\alpha}\right) & =0, \\
\frac{\partial S}{\partial q^{\alpha}}+H_{\alpha}\left(t, q^{i}, \frac{\partial S}{\partial q^{i}}, q^{\alpha}, \dot{q}^{\alpha}\right) & =0 .
\end{aligned}
$$

Now, on the direct product of $T Q_{n_{p}}^{*} \times T Q_{n-n_{p}}$, the action is

$$
S=\int\left(p_{i} d q^{i}-H_{\alpha} d q^{\alpha}-H_{0} d t\right) .
$$

Variation of (3.14) should be made independently on $2 n_{p}$-reduced canonical coordinates $q^{i}, p_{i}$ and $\left(n-n_{p}\right)$ noncanonical generalized coordinates $q^{\alpha}$. Under the assumption that the variations of $\delta q^{i}, \delta p_{i}, \delta q^{\alpha}$ at the upper and lower limits vanish after integration by parts for the variation of the action (3.14) we obtain

$$
\begin{aligned}
\delta S & =\int \delta p_{i}\left[d q^{i}-\frac{\partial H_{0}}{\partial p_{i}} d t-\frac{\partial H_{\beta}}{\partial p_{i}} d q^{\beta}\right]+\int \delta q^{i}\left[-d p_{i}-\frac{\partial H_{0}}{\partial q^{i}} d t-\frac{\partial H_{\beta}}{\partial q^{i}} d q^{\beta}\right]+ \\
& \int \delta q^{\alpha}\left[\frac{\partial H_{\alpha}}{\partial \dot{q}^{\beta}} d \dot{q}^{\beta}+\frac{\partial H_{\alpha}}{\partial q^{i}} d q^{i}+\frac{\partial H_{\alpha}}{\partial p_{i}} d p_{i}+\frac{d}{d t}\left(\frac{\partial H_{0}}{\partial \dot{q}^{\alpha}}+\frac{\partial H_{\beta}}{\partial \dot{q}^{\alpha}} \dot{q}^{\beta}\right) d t+\right. \\
& \left.+\left(\frac{\partial H_{\alpha}}{\partial q^{\beta}}-\frac{\partial H_{\beta}}{\partial q^{\alpha}}\right) d q^{\beta}+\left(\frac{\partial H_{\alpha}}{\partial t}-\frac{\partial H_{0}}{\partial q^{\alpha}}\right) d t\right]
\end{aligned}
$$

The equations of motion for the partial Hamiltonian formalism can be derived from the principle of the least action $\delta S=0$. Taking into account the fact that the variations of $\delta q^{i}$, 
$\delta p_{i}, \delta q^{\alpha}$ are independent, their coefficients (each bracket in (3.15) vanish. We introduce the (reduced) Poisson bracket for two functions $A$ and $B$ in the reduced phase space $T Q_{n_{p}}^{*}$

$$
\{A, B\}=\frac{\partial A}{\partial q^{i}} \frac{\partial B}{\partial p_{i}}-\frac{\partial B}{\partial q^{i}} \frac{\partial A}{\partial p_{i}} .
$$

Then, substituting $d q^{i}$ and $d p_{i}$ from the first row of (3.15) in the second line, we obtain the equations of motion on $T Q_{n_{p}}^{*} \times T Q_{n-n_{p}}$ in the differential form

$$
\begin{aligned}
d q^{i} & =\left\{q^{i}, H_{0}\right\} d t+\left\{q^{i}, H_{\beta}\right\} d q^{\beta}, \\
d p_{i} & =\left\{p_{i}, H_{0}\right\} d t+\left\{p_{i}, H_{\beta}\right\} d q^{\beta}, \\
\frac{\partial H_{\alpha}}{\partial \dot{q}^{\beta}} d \dot{q}^{\beta}+\frac{d}{d t}\left(\frac{\partial H_{0}}{\partial \dot{q}^{\alpha}}+\frac{\partial H_{\beta}}{\partial \dot{q}^{\alpha}} \dot{q}^{\beta}\right) d t & =\left(\frac{\partial H_{\beta}}{\partial q^{\alpha}}-\frac{\partial H_{\alpha}}{\partial q^{\beta}}+\left\{H_{\beta}, H_{\alpha}\right\}\right) d q^{\beta} \\
& +\left(\frac{\partial H_{0}}{\partial q^{\alpha}}-\frac{\partial H_{\alpha}}{\partial t}+\left\{H_{0}, H_{\alpha}\right\}\right) d t .
\end{aligned}
$$

We see that on $T Q_{n_{p}}^{*}$, we have the first-order equations (3.17)-(3.18) for the canonical coordinates $q^{i}, p_{i}$, as it should be (see (2.11), while on the (noncanonical) subspace $T Q_{n-n_{p}}$, the equation 3.19 is still of second order with respect to the noncanonical generalized coordinates $q^{\alpha}$, namely,

$$
\begin{aligned}
\dot{q}^{i} & =\left\{q^{i}, H_{0}\right\}+\left\{q^{i}, H_{\beta}\right\} \dot{q}^{\beta}, \\
\dot{p}_{i} & =\left\{p_{i}, H_{0}\right\}+\left\{p_{i}, H_{\beta}\right\} \dot{q}^{\beta}, \\
\frac{\partial H_{\alpha}}{\partial \dot{q}^{\beta}} \ddot{q}^{\beta}+\frac{d}{d t}\left(\frac{\partial H_{0}}{\partial \dot{q}^{\alpha}}+\frac{\partial H_{\beta}}{\partial \dot{q}^{\alpha}} \dot{q}^{\beta}\right) & =\left(\frac{\partial H_{\beta}}{\partial q^{\alpha}}-\frac{\partial H_{\alpha}}{\partial q^{\beta}}+\left\{H_{\beta}, H_{\alpha}\right\}\right) \dot{q}^{\beta} \\
+ & \left(\frac{\partial H_{0}}{\partial q^{\alpha}}-\frac{\partial H_{\alpha}}{\partial t}+\left\{H_{0}, H_{\alpha}\right\}\right) .
\end{aligned}
$$

It is important to note that the resulting system of equations of motion (3.20)-(3.22) of the partial Hamiltonian formalism is valid for any number of reduced momenta (which becomes a free parameter together with the number $\left(n-n_{p}\right)$ of the additional Hamiltonians $\left.H_{\alpha}\right)$

$$
0 \leq n_{p} \leq n
$$

In other words, the dynamics is independent of the dimension of the reduced phase space. In this case, the boundary values of $n_{p}$ correspond to the Lagrangian and Hamiltonian formalisms, respectively, so that we have the three cases, which are described by the equations (3.20)-(3.22):

(1) $n_{p}=0-$ the Lagrangian formalism on $T Q_{n}$ (we have only the last equation (3.22) without the Poisson brackets), and $\alpha=1, \ldots, n$;

(2) $0<n_{p}<n-$ the partial Hamiltonian formalism for $T Q_{n_{p}}^{*} \times T Q_{n-n_{p}}$ (all of the equations are considered);

(3) $n_{p}=n$ - the standard Hamiltonian formalism on $T Q_{n}^{*}$ (the first two equations $\sqrt{3.20}$ )-3.21 without the second terms containing noncanonical generalized velocities are 
considered), which coincides with the standard Hamiltonian equations (2.11), and $i=$ $1, \ldots, n$.

Let us show that in the case 1) we obtain the standard Lagrange equations (for the noncanonical variables $q^{\alpha}$ ). Indeed, the equation (3.22) without the Poisson brackets (the canonical variables $q^{i}, p_{i}$ are absent in the case $n_{p}=0$ ) can be rewritten as

$$
\frac{\partial H_{\alpha}}{\partial \dot{q}^{\beta}} \ddot{q}^{\beta}+\frac{d}{d t} \frac{\partial}{\partial \dot{q}^{\alpha}}\left(H_{0}+H_{\beta} \dot{q}^{\beta}\right)-\frac{d H_{\alpha}}{d t}=\frac{\partial}{\partial q^{\alpha}}\left(H_{0}+H_{\beta} \dot{q}^{\beta}\right)-\frac{\partial H_{\alpha}}{\partial q^{\beta}} \dot{q}^{\beta}-\frac{\partial H_{\alpha}}{\partial t},
$$

where we derive

$$
\begin{aligned}
\frac{d}{d t}\left(\frac{\partial H_{0}}{\partial \dot{q}^{\alpha}}+\frac{\partial H_{\beta}}{\partial \dot{q}^{\alpha}} \dot{q}^{\beta}\right) & =\frac{d}{d t}\left[\frac{\partial}{\partial \dot{q}^{\alpha}}\left(H_{0}+H_{\beta} \dot{q}^{\beta}\right)-H_{\beta} \frac{\partial}{\partial \dot{q}^{\alpha}} \dot{q}^{\beta}\right] \\
& =\frac{d}{d t}\left[\frac{\partial}{\partial \dot{q}^{\alpha}}\left(H_{0}+H_{\beta} \dot{q}^{\beta}\right)-H_{\alpha}\right]
\end{aligned}
$$

Using the expression for the total derivative of $d H_{\alpha} / d t$, we obtain from (3.24)

$$
\frac{d}{d t} \frac{\partial}{\partial \dot{q}^{\alpha}}\left(H_{0}+H_{\beta} \dot{q}^{\beta}\right)=\frac{\partial}{\partial q^{\alpha}}\left(H_{0}+H_{\beta} \dot{q}^{\beta}\right) .
$$

The formula to determine the partial Hamiltonian (3.5) without variables $q^{i}, p_{i}$, taking into account (3.7) is

$$
H_{0}=-H_{\alpha} \dot{q}^{\alpha}-L .
$$

Hence, $H_{0}+H_{\beta} \dot{q}^{\beta}=-L$, so that from (3.26), we obtain the standard Lagrange equations in noncanonical sector

$$
\frac{d}{d t} \frac{\partial}{\partial \dot{q}^{\alpha}} L=\frac{\partial}{\partial q^{\alpha}} L
$$

As in the standard Hamiltonian formalism [20], a non-trivial dynamics in the noncanonical sector is determined by the presence of noncanonical terms with second derivatives, that is, the presence of non-zero terms on the left and total time derivatives in the right side of (3.22).

Consider a special case of the partial Hamiltonian formalism, when these terms (with second derivatives) vanish, and call it nondynamical in the noncanonical sector. This requires the following conditions on the Hamiltonians

$$
\frac{\partial H_{0}}{\partial \dot{q}^{\beta}}=0, \quad \frac{\partial H_{\alpha}}{\partial \dot{q}^{\beta}}=0, \quad \alpha, \beta=n_{p}+1, \ldots, n .
$$

Then (3.22) will have only the right side, which can be written as

$$
\left(\frac{\partial H_{\beta}}{\partial q^{\alpha}}-\frac{\partial H_{\alpha}}{\partial q^{\beta}}+\left\{H_{\beta}, H_{\alpha}\right\}\right) \dot{q}^{\beta}=-\left(\frac{\partial H_{0}}{\partial q^{\alpha}}-\frac{\partial H_{\alpha}}{\partial t}+\left\{H_{0}, H_{\alpha}\right\}\right),
$$

which is a system of linear algebraic equations for the noncanonical velocities $\dot{q}^{\alpha}$ for given Hamiltonians $H_{0}, H_{\alpha}$. As in (3.30) there are no noncanonical accelerations $\ddot{q}^{\alpha}$, so on $T Q_{n-n_{p}}$ there is no real dynamics, if (3.29) is satisfied. 


\section{Singular theories}

Let us consider in more detail the conditions $(3.29)$ and express them in terms of the Lagrangian. Using (3.5) and the definition of the additional Hamiltonians (3.7), we obtain

$$
\frac{\partial^{2} L}{\partial \dot{q}^{\alpha} \partial \dot{q}^{\beta}}=0, \quad \alpha, \beta=n_{p}+1, \ldots, n .
$$

This means that the dynamics is described by a degenerate Lagrangian (singular) theory, so that the rank $r_{W}$ of the Hessian matrix

$$
W_{A B}=\left\|\frac{\partial^{2} L}{\partial \dot{q}^{A} \partial \dot{q}^{B}}\right\|, \quad A, B=1, \ldots, n
$$

is not only less than the dimension $n$ of the configuration space, but less than or equal to the number of momenta (due to 4.10)

$$
r_{W} \leq n_{p}
$$

In considering the strict inequality in (4.3) we find that the definition of "extra" $\left(n_{p}-r_{W}\right)$ momenta results in a $\left(n_{p}-r_{W}\right)$ relations, just as in the Dirac theory of constraints [7], where there are $\left(n-r_{W}\right)$ (primary) constraints, if the standard Hamiltonian formalism is used. It is important that the dimension $n$ of the configuration space and the rank of the Hessian $r_{W}$ are fixed by the problem statement initially, which does not allow a change to the number of constraints.

In the case of the partial Hamiltonian formalism the number $n_{p}$ is a free parameter that can be chosen so that the constraints do not appear at all. To do this, it is natural to equate the number of momenta with the rank of the Hessian

$$
n_{p}=r_{W} .
$$

As a result, the singular dynamics (theory with degenerate Lagrangians) can be formulated in such a way that constraints will not occur (primary, secondary or of higher level) [14 15]. To do this, first, rename the index of the Hessian matrix $W_{A B}$ (4.2) so that the non-singular minor rank $r_{W}$ is in the upper left hand corner, denote with the Latin alphabet $i, j$ the first $r_{W}$ indices and with Greek letters $\alpha, \beta$ the remaining $\left(n-r_{W}\right)$ indices. Next, we write the equations of motion (3.20)-(3.21), (3.30) as

$$
\begin{aligned}
& \dot{q}^{i}=\left\{q^{i}, H_{0}\right\}+\left\{q^{i}, H_{\beta}\right\} \dot{q}^{\beta}, \\
& \dot{p}_{i}=\left\{p_{i}, H_{0}\right\}+\left\{p_{i}, H_{\beta}\right\} \dot{q}^{\beta}, \\
& F_{\alpha \beta} \dot{q}^{\beta}=G_{\alpha},
\end{aligned}
$$

where the index values are connected with the rank of the Hessian by $i=1, \ldots, r_{W}$, $\alpha, \beta=r_{W}+1, \ldots, n$, and

$$
\begin{aligned}
F_{\alpha \beta} & =\frac{\partial H_{\alpha}}{\partial q^{\beta}}-\frac{\partial H_{\beta}}{\partial q^{\alpha}}+\left\{H_{\alpha}, H_{\beta}\right\} \\
G_{\alpha} & =D_{\alpha} H_{0}=\frac{\partial H_{0}}{\partial q^{\alpha}}-\frac{\partial H_{\alpha}}{\partial t}+\left\{H_{0}, H_{\alpha}\right\}
\end{aligned}
$$


Note that the system of equations (4.5)-(4.9) coincides with the equations derived in the approach to singular theories, which uses the mixed solutions of Clairaut's equation [14]15] (except for the term with the partial time derivative of $H_{\alpha}$ in (4.9)). Equations (4.5)-(4.7) are a system of first order differential equations for the canonical coordinates $q^{i}, p_{i}$, while with respect to the noncanonical velocities $\dot{q}^{\alpha}$, this is an algebraic system. Indeed, (4.7) is the usual system of linear equations with respect to $\dot{q}^{\alpha}$, and the properties of its solutions can be used to classify classical singular theories. We will consider only those cases, when the system (4.7) is consistent, then there are two possibilities determined by the rank of the matrix $F_{\alpha \beta}$ :

(1) Nongauge theory, when $\operatorname{rank} F_{\alpha \beta}=r_{F}=n-r_{W}$ is full, so that the matrix $F_{\alpha \beta}$ is invertible. Then from (4.7) we can determine all noncanonical velocities by

$$
\dot{q}^{\alpha}=\bar{F}^{\alpha \beta} G_{\beta},
$$

where $\bar{F}^{\alpha \beta}$ is the inverse matrix of $F_{\alpha \beta}$, defined by the equation $\bar{F}^{\alpha \beta} F_{\beta \gamma}=$ $F_{\gamma \beta} \bar{F}^{\beta \alpha}=\delta_{\gamma}^{\alpha}$.

(2) Gauge theory, the rank of the $F_{\alpha \beta}$ is incomplete, that is, $r_{F}<n-r_{W}$, and the matrix $F_{\alpha \beta}$ is noninvertible. In this case, we can find from (4.7) only $r_{F}$ noncanonical velocities, while the rest $\left(n-r_{W}-r_{F}\right)$ of the velocities remain arbitrary gauge parameters that correspond to the symmetries of a singular dynamical system. In the particular case $r_{F}=0$ (or zero matrix $F_{\alpha \beta}$ ) from (4.7) we obtain

$$
G_{\alpha}=0,
$$

and all the noncanonical velocities correspond to $\left(n-r_{W}\right)$ gauge parameters of the theory.

In the first case (nongauge theory) all noncanonical velocities can be excluded by (4.10) and substituting them in (4.5)-(4.6). Then we obtain the Hamilton-like equations for nongauge singular systems

$$
\begin{aligned}
& \dot{q}^{i}=\left\{q^{i}, H_{0}\right\}_{\text {nongauge }}, \\
& \dot{p}_{i}=\left\{p_{i}, H_{0}\right\}_{\text {nongauge }},
\end{aligned}
$$

where we have introduced a new (nongauge) bracket for the two dynamical quantities $A, B$

$$
\{A, B\}_{\text {nongauge }}=\{A, B\}+D_{\alpha} A \cdot \bar{F}^{\alpha \beta} \cdot D_{\beta} B,
$$

and $D_{\alpha}$ is defined in (4.9). From (4.12)-(4.13), it follows that the new nongauge bracket (4.14) uniquely determines the evolution of any dynamical quantity $A$ in time

$$
\frac{d A}{d t}=\frac{\partial A}{\partial t}+\left\{A, H_{0}\right\}_{\text {nongauge }} .
$$

It is important too that the nongauge bracket (4.14) has all the properties of the Poisson bracket: it is antisymmetric and satisfies the Jacobi identity. Therefore, the definition (4.14) can be considered as some deformation of the Poisson bracket, but not for all $2 n$ variables as in the standard case, but only for $2 r_{W}$ canonical coordinates $\left(q^{i}, p_{i}\right), i=1, \ldots, r_{W}$. It 
follows from (4.14) and (4.15) that, as in the standard case, if $H_{0}$ does not depend on time explicitly, then it is conserved.

In the second case (gauge theory), only some of the noncanonical velocities $\dot{q}^{\alpha}$ can be eliminated, the number of which is equal to the rank $r_{F}$ of the matrix $F_{\alpha \beta}$, and the rest $\left(n-r_{W}-r_{F}\right)$ of the velocities are still arbitrary and can serve as gauge parameters. Indeed, if the matrix $F_{\alpha \beta}$ is singular and of the rank $r_{F}$, then we can bring it to the form that a non-singular minor of size $r_{F} \times r_{F}$ which will be in the upper left-hand corner. Then in the system (4.7), only the first $r_{F}$ equations are independent. Let us present ("noncanonical") indices $\alpha, \beta=r_{W}+1, \ldots, n$ in the form of pairs $\left(\alpha_{1}, \alpha_{2}\right),\left(\beta_{1}, \beta_{2}\right)$, where $\alpha_{1}, \beta_{1}=$ $r_{W}+1, \ldots, r_{F}$ number the first $r_{F}$ independent rows of the matrix $F_{\alpha \beta}$ and correspond to the nonsingular minor of $F_{\alpha_{1} \beta_{1}}$, the remaining $\left(n-r_{W}-r_{F}\right)$ rows will be dependent on the first ones, and $\alpha_{2}, \beta_{2}=r_{F}+1, \ldots, n$. Then the system (4.7) can be written as

$$
\begin{aligned}
& F_{\alpha_{1} \beta_{1}} \dot{q}^{\beta_{1}}+F_{\alpha_{1} \beta_{2}} \dot{q}^{\beta_{2}}=G_{\alpha_{1}}, \\
& F_{\alpha_{2} \beta_{1}} \dot{q}^{\beta_{1}}+F_{\alpha_{2} \beta_{2}} \dot{q}^{\beta_{2}}=G_{\alpha_{2}} .
\end{aligned}
$$

Since $F_{\alpha_{1} \beta_{1}}$ is non-singular by construction, we can express the first $r_{F}$ canonical velocities $\dot{q}^{\alpha_{1}}$ in terms of the remaining $\left(n-r_{W}-r_{F}\right)$ velocities $\dot{q}^{\alpha_{2}}$ as follows

$$
\dot{q}^{\alpha_{1}}=\bar{F}^{\alpha_{1} \beta_{1}} G_{\beta_{1}}-\bar{F}^{\alpha_{1} \beta_{1}} F_{\beta_{1} \alpha_{2}} \dot{q}^{\alpha_{2}},
$$

where $\bar{F}^{\alpha_{1} \beta_{1}}$ is $r_{F} \times r_{F}$-matrix which is inverse to $F_{\alpha_{1} \beta_{1}}$. Further, due to the fact that $\operatorname{rank} F_{\alpha_{1} \beta_{1}}=r_{F}$, the other blocks can be expressed via a non-singular block $F_{\alpha_{1} \beta_{1}}$

$$
\begin{aligned}
F_{\alpha_{2} \beta_{1}} & =\lambda_{\alpha_{2}}^{\alpha_{1}} F_{\alpha_{1} \beta_{1}}, \\
F_{\alpha_{2} \beta_{2}} & =\lambda_{\alpha_{2}}^{\alpha_{1}} F_{\alpha_{1} \beta_{2}}=\lambda_{\alpha_{2}}^{\alpha_{1}} \lambda_{\beta_{2}}^{\gamma_{1}} F_{\alpha_{1} \gamma_{1}}, \\
G_{\alpha_{2}} & =\lambda_{\alpha_{2}}^{\alpha_{1}} G_{\alpha_{1}},
\end{aligned}
$$

where $\lambda_{\alpha_{2}}^{\alpha_{1}}=\lambda_{\alpha_{2}}^{\alpha_{1}}\left(q^{i}, p_{i}, q^{\alpha}\right)$ are $r_{F} \times\left(n-r_{W}-r_{F}\right)$ smooth functions. Since the matrix $F_{\alpha \beta}$ is given, we can determine the function $\lambda_{\alpha_{2}}^{\alpha_{1}}$ by $r_{F} \times\left(n-r_{W}-r_{F}\right)$ equations (4.19)

$$
\lambda_{\alpha_{2}}^{\alpha_{1}}=F_{\alpha_{2} \beta_{1}} \bar{F}^{\alpha_{1} \beta_{1}} .
$$

Because $\left(n-r_{W}-r_{F}\right)$ velocities $\dot{q}^{\alpha_{2}}$ are arbitrary, we can put them equal to zero

$$
\dot{q}^{\alpha_{2}}=0, \quad \alpha_{2}=r_{F}+1, \ldots, n,
$$

which can be considered as a gauge condition. Then from (4.18), it follows that

$$
\dot{q}^{\alpha_{1}}=\bar{F}^{\alpha_{1} \beta_{1}} G_{\beta_{1}}, \quad \alpha_{1}=r_{W}+1, \ldots, r_{F} .
$$

By analogy with 4.14), we introduce a new (gauge) bracket

$$
\{A, B\}_{\text {gauge }}=\{A, B\}+D_{\alpha_{1}} A \cdot \bar{F}^{\alpha_{1} \beta_{1}} \cdot D_{\beta_{1}} B .
$$

Then the equations of motion (4.5)-4.7) can be written in the Hamiltonian-like form (as (4.12)-(4.13))

$$
\begin{aligned}
& \dot{q}^{i}=\left\{q^{i}, H_{0}\right\}_{\text {gauge }}, \\
& \dot{p}_{i}=\left\{p_{i}, H_{0}\right\}_{\text {gauge }} .
\end{aligned}
$$


The evolution of a physical quantity $A$ in time, as 4.15 , is determined by the gauge bracket (4.25)

$$
\frac{d A}{d t}=\frac{\partial A}{\partial t}+\left\{A, H_{0}\right\}_{\text {gauge }} .
$$

In the particular case, when $r_{F}=0$, we have

$$
F_{\alpha \beta}=0,
$$

and hence all additional Hamiltonians vanish $H_{\alpha}=0$, then it is seen from the definition (3.7) that the Lagrangian does not depend on the noncanonical velocities $\dot{q}^{\alpha}$. Therefore, taking into account (4.29) and (4.7) we find that the partial Hamiltonian $H_{0}$ does not depend on the noncanonical generalized coordinates $q^{\alpha}$

$$
\frac{\partial H_{0}}{\partial q^{\alpha}}=0
$$

if $H_{0}$ is manifestly independent of time. In this case, the gauge bracket coincides with the Poisson bracket, since the second term in (4.25) vanishes. Thus, we have shown that the singular theories (with degenerate Lagrangians) at the classical level can be described in terms of the partial Hamiltonian formalism with the number of momenta of $n_{p}$, equal to the rank $r_{W}$ of the Hessian matrix $n_{p}=r_{W}$, without the introduction of additional relationships between the dynamical variables (constraints).

\section{Origin of constraints in singular theories}

As noted above (after (4.3)), the introduction of additional dynamical variables must necessarily give rise to additional relationships between them. For example, let us consider the "extra" $\left(n-r_{W}\right)$ momenta $p_{\alpha}$ (since we have a complete description of the dynamics without them), which correspond to noncanonical generalized velocities $\dot{q}^{\alpha}$ for the standard definition [7]

$$
p_{\alpha}=\frac{\partial L}{\partial \dot{q}^{\alpha}}, \quad \alpha=r_{W}+1, \ldots, n .
$$

So (5.1), together with the definition of the partial generalized canonical momenta (3.4) coincide with the standard definition of the "full" momenta (2.5). Using the definition of additional Hamiltonians (3.7), we get the same $\left(n-r_{W}\right)$ relations

$$
\Phi_{\alpha}=p_{\alpha}+H_{\alpha}=0, \quad \alpha=r_{W}+1, \ldots, n,
$$

which are called the (primary) constraints [7] (in a resolved form). These relations (5.2) are similar to the standard procedure of extension of the phase space (3.2). One can enter any number $n_{p}^{(a d d)}$ of "extra" momenta $0 \leq n_{p}^{(a d d)} \leq n-r_{W}$, then the theory will have the same number $n_{p}^{(a d d)}$ of (primary) constraints. In the partial Hamiltonian formalism we have considered the case of $n_{p}^{(a d d)}=0$, while in the Dirac theory, $n_{p}^{(a d d)}=n-r_{W}$, although it is possible to take intermediate variants, to solve a specific task.

Now, the transition to the Hamiltonian by the standard formula

$$
H_{\text {total }}=p_{i} \dot{q}^{i}+p_{\alpha} \dot{q}^{\alpha}-L
$$


cannot be done directly), because it is impossible to express the noncanonical velocities $\dot{q}^{\alpha}$ through the "extra" momenta $p_{\alpha}$ and then apply the standard Legendre transformation. But it is possible to transform $H_{\text {total }}$ (5.3) in such a way that one can use the method of undetermined coefficients [7]. It is important that the constraints $\Phi_{\alpha}$ do not depend on the noncanonical generalized velocities $\dot{q}^{\alpha}$, as well as the Hamiltonians $H_{0}, H_{\alpha}$, because the rank of the Hessian matrix is equal to $r_{W}$. So the total Hamiltonian can be written as

$$
H_{\text {total }}=H_{0}+\dot{q}^{\alpha} \Phi_{\alpha},
$$

where $\dot{q}^{\alpha}$ play the role of undetermined coefficients. The equations of motion can be written in a Hamiltonian-like form in terms of the total Hamiltonian and the full Poisson bracket (2.12) as follows

$$
\begin{aligned}
& d q^{A}=\left\{q^{A}, H_{\text {total }}\right\}_{\text {full }} d t, \\
& d p_{A}=\left\{p_{A}, H_{\text {total }}\right\}_{\text {full }} d t
\end{aligned}
$$

with the $\left(n-r_{W}\right)$ of additional conditions (5.2). However, the equations (5.5)-(5.6) and (5.2) are not sufficient to solve the problem: to find the equations for the undetermined coefficients $\dot{q}^{\alpha}$ in (5.4). These equations can be derived from some additional principles, such as conservation relations (5.2) in time [7]

$$
\frac{d \Phi_{\alpha}}{d t}=0 .
$$

The time dependence of any physical quantity $A$ is now determined by the total Hamiltonian and the full Poisson bracket

$$
\frac{d A}{d t}=\frac{\partial A}{\partial t}+\left\{A, H_{t o t a l}\right\}_{\text {full }} .
$$

If the constraints do not depend explicitly on time, then from (5.8) and (5.4) we obtain

$$
\left\{\Phi_{\alpha}, H_{\text {total }}\right\}_{\text {full }}=\left\{\Phi_{\alpha}, H_{0}\right\}_{\text {full }}+\left\{\Phi_{\alpha}, \Phi_{\beta}\right\}_{\text {full }} \dot{q}^{\beta}=0
$$

which is a system of equations for the undetermined coefficients $\dot{q}^{\alpha}$. Note that (5.9) coincides with 4.7), because

$$
\begin{aligned}
F_{\alpha \beta} & =\left\{\Phi_{\alpha}, \Phi_{\beta}\right\}_{\text {full }}, \\
D_{\alpha} H_{0} & =\left\{\Phi_{\alpha}, H_{0}\right\}_{\text {full }} .
\end{aligned}
$$

However, in contrast to the reduced description (without the $\left(n-r_{W}\right)$ "extra" momenta $\left.p_{\alpha}\right)$, where (4.7) is a system of $\left(n-r_{W}\right)$ linear equations in $\left(n-r_{W}\right)$ unknowns $\dot{q}^{\alpha}$, the extended system (5.9) can lead to additional constraints (of higher stages), which significantly complicates the analysis of the physical dynamics [7, 10].

It follows from (5.10)-(5.11), that the new brackets (gauge (4.25) and nongauge (4.14) transform into the corresponding Dirac brackets. We also note that our classification on the gauge and nongauge theories corresponds to the first- and second-class constraints [7], and the limiting case of $F_{\alpha \beta}=0$ (4.29) corresponds to Abelian constraints [23, 24].

cTherefore, $H_{\text {total }}$ is also not a "true" Hamiltonian, as $H_{0}$ in 3.5 . 


\section{Conclusion}

Thus, in this paper a "shortened" formulation of classical singular theories is given. In it there is no concept of constraints, because "extra" dynamical variables, namely, the generalized momenta corresponding to noncanonical coordinates, are not introduced. To this purpose, a partial Hamiltonian formalism is proposed. It is shown that a special case of it effectively describes multi-time dynamics. It is proved that singular theories (with degenerate Lagrangians) can be described in the framework of our approach without the introduction of additional relations between the dynamical variables (constraints), if the number of canonical generalized momenta coincides with the rank of the Hessian matrix $n_{p}=r_{W}$. From a physical point of view, the introduction of the "extra" momenta is not necessary, because there is no dynamics in these (degenerate) directions.

The Hamiltonian formulation of singular theories is done by introducing new brackets (gauge (4.25) and nongauge (4.14), which have all the properties of the Poisson brackets (antisymmetry, Jacobi identity and their appearance in the equations of motion and evolution of the system in time).

If one extends the phase space to the full phase space, these brackets become the Dirac brackets, and constraints are imposed on the "extra" momenta.

Our analysis suggests that the quantization of singular systems under the proposed "shortened" approach can be carried out in a standard way, while not all $2 n$ variables of the extended phase space will be quantized, but only $2 r_{W}$ variables of the (initially) reduced phase space. The remaining (noncanonical) variables can be treated as continuous parameters.

\section{Acknowledgments}

The author is grateful to I. H. Brevik, G. A. Goldin, U. Günther, M. Hewitt, I. Kanatchikov, G. Ch. Kurinnoy, A. Nurmagambetov, A. A. Voronov and M. Walker for useful discussions. He would like to express deep thankfulness to Jim Stasheff for carefully reading all versions and making many corrections and important remarks, and to S. Deser for pointing out [21] reprinted as arXiv:gr-qc/0405109. Also, the author is thankful to the Alexander von Humboldt Foundation for funding his research stay at the University of Münster, and J. Cuntz for kind hospitality.

\section{A. Appendix: Singular theory as multi-time dynamics}

The condition (3.29) means that the Hamiltonians do not depend explicitly on the noncanonical velocities $\dot{q}^{\alpha}$, that is, $H_{0}=H_{0}\left(t, q^{i}, p_{i}, q^{\alpha}\right), H_{\alpha}=H_{\alpha}\left(t, q^{i}, p_{i}, q^{\alpha}\right), \alpha=$ $n_{p}+1, \ldots, n$. Thus, the dynamical problem is defined on the manifold $T Q_{n_{p}}^{*} \times Q_{n-n_{p}}$, so that $q^{\alpha}$ actually play the role of real parameters, similar to the timed.

\footnotetext{
${ }^{\mathrm{d}}$ In this case the nondynamical $Q_{n-n_{p}}$ is isomorphic to the real space $R^{n-n_{p}}$.
} 
Recalling (3.1) and reversing it, we can interpret $\left(n-n_{p}\right)$ canonical generalized coordinates $q^{\alpha}$ as $\left(n-n_{p}\right)$ "extra" (to $\left.t\right)$ times, and $H_{\alpha}$ as $\left(n-n_{p}\right)$ corresponding Hamiltonians. Indeed, we introduce the notation

$$
\begin{aligned}
\tau^{\mu} & =t, \quad \mathbf{H}_{\mu}=H_{0}, \quad \mu=0, \\
\tau^{\mu} & =q^{\mu+n_{p}}, \quad \mathbf{H}_{\mu}=H_{\mu+n_{p}}, \quad \mu=1, \ldots,\left(n-n_{p}\right),
\end{aligned}
$$

where $\mathrm{H}_{\mu}=\mathrm{H}_{\mu}\left(\tau_{\mu}, q^{i}, p_{i}\right)$ are Hamiltonians of the multi-time dynamics with $n_{\mu}=n-$ $n_{p}+1$ times $\tau^{\mu}$. Note that $\tau^{\mu}$ can be called generalized times, because they are not real times (for the real multi-time physics see [25, 26] and review [27]), in the same way as generalized coordinates have nothing to do with space-time coordinates [20].

In this formulation, the differential of the action $\mathrm{S}=\mathrm{S}\left(\tau^{\mu}, q^{i}\right)$ in the multi-time dynamics can be written as [28] (see also [21])

$$
d \mathrm{~S}=p_{i} d q^{i}-\mathrm{H}_{\mu} d \tau^{\mu}, \quad i=1, \ldots, n_{p}, \quad \mu=0, \ldots,\left(n-n_{p}\right)
$$

It follows that the partial derivatives of the action are

$$
\frac{\partial \mathrm{S}}{\partial q^{i}}=p_{i}, \quad \frac{\partial \mathrm{S}}{\partial \tau^{\mu}}=-\mathrm{H}_{\mu},
$$

and the system of $\left(n-n_{p}+1\right)$ Hamilton-Jacobi equations is

$$
\frac{\partial \mathrm{S}}{\partial \tau^{\mu}}+\mathrm{H}_{\mu}\left(\tau_{\mu}, q^{i}, \frac{\partial \mathrm{S}}{\partial q^{i}}\right)=0, \quad \mu=0, \ldots,\left(n-n_{p}\right) .
$$

We note that from (2.1) and (A.4) it follows the relation between $\mathrm{H}_{\mu}$. Indeed, we differentiate the Hamilton-Jacobi equations (2.1) on $\tau^{\nu}$

$$
\begin{aligned}
\frac{\partial^{2} \mathbf{S}}{\partial \tau^{\mu} \partial \tau^{\nu}} & =-\frac{\partial \mathbf{H}_{\mu}}{\partial \tau^{\nu}}-\frac{\partial \mathbf{H}_{\mu}}{\partial p_{i}} \frac{\partial}{\partial \tau^{\nu}} \frac{\partial \mathrm{S}}{\partial q^{i}}=-\frac{\partial \mathbf{H}_{\mu}}{\partial \tau^{\nu}}-\frac{\partial \mathbf{H}_{\mu}}{\partial p_{i}}\left(-\frac{\partial \mathbf{H}_{\nu}}{\partial q^{i}}-\frac{\partial \mathbf{H}_{\nu}}{\partial p_{j}} \frac{\partial}{\partial q^{i}} \frac{\partial \mathrm{S}}{\partial q^{j}}\right) \\
& =-\frac{\partial \mathbf{H}_{\mu}}{\partial \tau^{\nu}}+\frac{\partial \mathbf{H}_{\mu}}{\partial p_{i}} \frac{\partial \mathbf{H}_{\nu}}{\partial q^{i}}+\frac{\partial \mathbf{H}_{\mu}}{\partial p_{i}} \frac{\partial \mathbf{H}_{\nu}}{\partial p_{j}} \frac{\partial^{2} \mathrm{~S}}{\partial q^{i} \partial q^{j}} .
\end{aligned}
$$

Then antisymmetrization (A.6) gives the integrability condition

$$
\frac{\partial^{2} \mathbf{S}}{\partial \tau^{\nu} \partial \tau^{\mu}}-\frac{\partial^{2} \mathbf{S}}{\partial \tau^{\mu} \partial \tau^{\nu}}=\frac{\partial \mathbf{H}_{\mu}}{\partial \tau^{\nu}}-\frac{\partial \mathbf{H}_{\nu}}{\partial \tau^{\mu}}+\left\{\mathbf{H}_{\mu}, \mathbf{H}_{\nu}\right\}=0 .
$$

To obtain the equations of motion, one needs to set the variation to zero: $\delta \mathrm{S}=0$, where

$$
\mathrm{S}=\int\left(p_{i} d q^{i}-\mathrm{H}_{\mu} d \tau^{\mu}\right)
$$

taking into account that independent variations $\delta q^{i}, \delta p_{i}, \delta \tau^{\mu}$ vanish at the ends of the interval of integration. We obtain

$$
\delta \mathrm{S}=\int \delta p_{i}\left(d q^{i}-\frac{\partial \mathrm{H}_{\mu}}{\partial p_{i}} d \tau^{\mu}\right)+\int \delta q^{i}\left(-d p_{i}-\frac{\partial \mathrm{H}_{\mu}}{\partial q^{i}} d \tau^{\mu}\right),
$$

where the equations of the Hamiltonian for the multi-time dynamics can be written in differential form [28]

$$
\begin{aligned}
d q^{i} & =\left\{q^{i}, \mathbf{H}_{\mu}\right\} d \tau^{\mu}, \\
d p_{i} & =\left\{p_{i}, \mathbf{H}_{\mu}\right\} d \tau^{\mu},
\end{aligned}
$$


which coincide with (3.17)-(3.18) by construction. The integrability conditions (A.7) can be also written in differential forme

$$
\left(\frac{\partial \mathbf{H}_{\mu}}{\partial \tau^{\nu}}-\frac{\partial \mathbf{H}_{\nu}}{\partial \tau^{\mu}}+\left\{\mathrm{H}_{\mu}, \mathrm{H}_{\nu}\right\}\right) d \tau^{\nu}=0, \quad \mu, \nu=0, \ldots,\left(n-n_{p}\right)
$$

which coincide with the equations (3.30), also written in differential form.

Thus, we have shown that the nondynamical sector in the noncanonical version of the partial Hamiltonian formalism (which is determined by the equations of motion (3.20)(3.22) with the additional integrability conditions (3.29), but without any conditions on the number of moments $n_{p}$ ) can be formulated as the multi-time dynamics with the number of generalized times (and corresponding Hamiltonians $\mathrm{H}_{\mu}$ ) $n_{\mu}=n-n_{p}+1$ and the equations A.10 - A.12. In this formulation the number of generalized times $n_{\mu}$ is not fixed, and $1 \leq n_{\mu} \leq n+1$, because the number of generalized momenta $n_{p}$ is arbitrary less than or equal to the dimension of the configuration space $n$. They are connected by the relation

$$
n_{\mu}+n_{p}=n+1
$$

which can be called a times-momenta rule. In the particular case of singular theories (with degenerate Lagrangians), the number of momenta $n_{p}$ is fixed by the condition (4.4) $n_{p}=$ $r_{W}$. So from (A.13) we obtain

$$
n_{\mu}+r_{W}=n+1,
$$

which can be called a times-rank rule. If (3.29) and A.14) are fulfilled, then such a singular theory can be (effectively) described by the multi-time dynamics.

\section{References}

[1] S. Weinberg, The Quantum Theory of Fields. Vols. 1,2,3 (Cambridge University Press, Cambridge, 1995-2000).

[2] J. F. Carinena, Theory of singular Lagrangians, Fortsch. Physik 38 (1990), 641-679.

[3] W. M. Tulczyjew, The Legendre transformation, Ann. Inst. Henri Poincaré A27 (1977), 101114.

[4] W. M. Tulczyjew and P. Urbański, A slow and careful Legendre transformation for singular Lagrangians, Acta Phys. Pol. B30 (1999), 2909-2977.

[5] M. R. Menzo and W. M. Tulczyjew, Infinitesimal symplectic relations and generalized Hamiltonian dynamics, Ann. Inst. Henri Poincaré A4 (1978), 349-367.

[6] G. Marmo, G. Mendella, and W. Tulczyjew, Constrained Hamiltonian systems as implicit differential equations, J. Phys. A30 (1997), 277-293.

[7] P. A. M. Dirac, Lectures on Quantum Mechanics (Yeshiva University, New York, 1964).

[8] D. M. Gitman and I. V. Tyutin, Canonical quantization of constrained fields (Nauka, M., 1986).

[9] M. Henneaux and C. Teitelboim, Quantization of Gauge Systems (Princeton University Press, Princeton, 1994).

[10] K. Sundermeyer, Constrained Dynamics (Springer-Verlag, Berlin, 1982).

${ }^{\mathrm{e}}$ As in the standard case, [16], the equations [A.10)A.3. 
[11] T. Regge and C. Teitelboim, Constrained Hamiltonian Systems (Academia Nazionale dei Lincei, Rome, 1976).

[12] J. M. Pons, On Dirac's incomplete analysis of gauge transformations, Stud. Hist. Philos. Mod. Phys. 36 (2005), 491-518.

[13] O. Mišković and J. Zanelli, Dynamical structure of irregular constrained systems, J. Math. Phys. 44 (2003), 3876-3887.

[14] S. Duplij, Generalized duality, Hamiltonian formalism and new brackets, J. Math. Physics, Analysis, Geometry 10 (2014), 189-220.

[15] S. Duplij, A new Hamiltonian formalism for singular Lagrangian theories, J. Kharkov National Univ., ser. Nuclei, Particles and Fields 969 (2011), 34-39.

[16] V. I. Arnold, Mathematical methods of classical mechanics (Springer, Berlin, 1989).

[17] T. Nakamura and S. Hamamoto, Higher derivatives and canonical formalisms, Progr. Theor. Phys. 95 (1996), 469-484.

[18] K. Andrzejewski, J. Gonera, P. Machalski, and P. Maślanka, Modified Hamiltonian formalism for higher-derivative theories, Phys. Rev. D82 (2010), 045008.

[19] C. Lanczos, The variational principles of mechanics, Second edition (Univ. Toronto Press, Toronto, 1962).

[20] L. D. Landau and E. M. Lifshitz, Mechanics (Pergamon Press, Oxford, 1969).

[21] R. Arnowitt, S. Deser, and C. W. Misner, The dynamics of General Relativity, in Gravitation: An Introduction To Current Research, ed. L. Witten (Wiley, New York, 1962), pp. 227-264.

[22] S. Duplij, Partial Hamiltonian formalism, multi-time dynamics and singular theories, $J$. Kharkov National Univ., ser. Nuclei, Particles and Fields 1059 (2013), 10-21.

[23] S. A. Gogilidze, A. M. Khvedelidze, and V. N. Pervushin, On Abelianization of first class constraints, J. Math. Phys. 37 (1996), 1760-1771.

[24] F. Loran, Non-Abelianizable first class constraints, Commun. Math. Phys. 254 (2005), 167178.

[25] J. Dorling, The dimensionality of time, Am. J. Phys. 38 (1970), 539-540.

[26] G. Dvali, G. Gabadadze, and G. Senjanovic, Constraints on extra time dimensions, in The Many Faces of the Superworld: Yuri Golfand Memorial Volume, ed. M. A. Shifman (World Scientific, Singapore, 2000).

[27] I. Bars and J. Terning, Why higher space or time dimensions?, in Extra Dimensions in Space and Time, ed. F. Nekoogar (Springer, Heidelberg, 2010), p. 218.

[28] G. Longhi, L. Lusanna, and J. M. Pons, On the many-time formulation of classical particle dynamics, J. Math. Phys. 30 (1989), 1893-1912. 